Funding Open Access funding provided by Projekt DEAL.

Open Access Dieser Artikel wird unter der Creative Commons Namensnennung 4.0 International Lizenz veröffentlicht, welche die Nutzung, Vervielfältigung, Bearbeitung, Verbreitung und Wiedergabe in jeglichem Medium und Format erlaubt, sofern Sie den/die ursprünglichen Autor(en) und die Quelle ordnungsgemäß nennen, einen Link zur Creative Commons Lizenz beifügen und angeben, ob Änderungen vorgenommen wurden.

Die in diesem Artikel enthaltenen Bilder und sonstiges Drittmaterial unterliegen ebenfalls der genannten Creative Commons Lizenz, sofern sich aus der Abbildungslegende nichts anderes ergibt. Sofern das betreffende Material nicht unter der genannten Creative Commons Lizenz steht und die betreffende Handlung nicht nach gesetzlichen Vorschriften erlaubt ist, ist für die oben aufgeführten Weiterverwendungen des Materials die Einwilligung des jeweiligen Rechteinhabers einzuholen.

Weitere Details zur Lizenz entnehmen Sie bitte der Lizenzinformation auf http://creativecommons.org/ licenses/by/4.0/deed.de.

\title{
Löschenkohl, Birte: Freiheit und Wiederholung. Politisches Handeln ohne Zukunft, 236 S., Fink, München/Paderborn 2018.
}

\author{
Matthias Gsänger \\ Online publiziert: 20. April 2020 \\ (C) Der/die Autor(en) 2020
}

Die Schwierigkeiten, mittels zielorientierten politischen Handelns bestimmte gesellschaftliche Zustände zu erreichen, waren schon Gegenstand der Steuerungsdebatte Anfang der 1990er Jahre. Dass die Probleme nicht kleiner werden, wenn es statt um policies, wie dem Ausbau von Kita-Plätzen, innerhalb einer bestehenden politischen Ordnung um grundlegende Veränderungen dieser Ordnung selbst geht, ist ein Ausgangspunkt von Birte Löschenkohls Buch. Ein anderer ist die Unplausibilität sowohl der Annahme souveränen Handelns als auch der Vorstellung einer quasi automatischen Entwicklung der Menschheit zum Besseren. Die Autorin sieht die Notwendigkeit, den Charakter von Praxisvollzügen umzudenken, und unterbreitet in ihrer Argumentation den Vorschlag, ,,das Potential der Wiederholung für die Entwicklung einer solchen konzeptuellen Alternative zu erkunden“ (S. 11). Sie unterscheidet zwischen

\footnotetext{
M. Gsänger $(\bowtie)$

Universität Würzburg, Würzburg, Deutschland

E-Mail: matthias.gsaenger@uni-wuerzburg.de
} 
konservativen, normierenden und öffnenden befreienden Aspekten von Wiederholungen. In der Verschränkung beider Momente liegt ihrer These nach das Potenzial der Wiederholung, ,Widerstand und Veränderung [...] auch unter eminent ungünstigen Umständen [...] zu konzipieren“ (S. 15). Die vier zentralen Kapitel des Buches widmet die Autorin der inspirierten und inspirierenden Rekonstruktion und Deutung von Texten Karl Marx', Sigmund Freuds, Friedrich Nietzsches und Sören Kierkegaards, bei denen sie, trotz aller Unterschiede, ein gemeinsames Interesse ausmacht. Alle vier Autoren skizzierten demnach Überlegungen zu einem Handeln, ,,was eine gegebene Ordnung, die sich verfestigt und gegenüber unserem Tun verselbständigt hat, zu überschreiten, unterwandern, oder anderweitig zu verändern sucht“" (S. 2).

In ihrem Schlusskapitel systematisiert Löschenkohl die von ihr herausgearbeiteten Blickwinkel der Klassiker auf das Thema Wiederholung in einer Typologie. Als (W I) „Macht der Wiederholung“ lassen sich demnach mit Bezug auf Marx und Michel Foucault Strukturen bezeichnen, die bestimmte Handlungsweisen fordern und hervorbringen (S. 206). Andere Handlungsweisen werden dadurch ausgeschlossen. Dieses Ausgeschlossene kann nun, begrifflich an Freud orientiert, in einem ,Symptomatischen Wiederholungszwang" (W II) wieder auftauchen. Es handelt sich hierbei um das „Drängen des Unmöglichen oder Ausgeschlossenen“, „dessen, was nicht sein darf" in die bestehende Ordnung hinein. Bei den Typen „Wiederholung der Wiederholung“ (W III) und „Wiederholung des Unmöglichen“ (W IV) handelt es sich in der Formulierung der Autorin um eng verschränkte „Handlungsvollzüge, die Vorschläge dazu sind, wie dem Problem des Verlusts von Handlungsperspektiven [...] begegnet werden kann. Dann bestünde die einzig gangbare Option darin, sich der Macht der Wiederholung nicht entgegenzustellen, sondern anders zu wiederholen“ (S. 211), Bestehendes zu modifizieren und zu verschieben, ohne Wissen, ,was genau das eigentlich Gewollte ist“" (S. 213). Aus dem wiederholt verfehlten Versuch, sich mittels von (W III) als kritischer Abstoßbewegung von dem Bestehenden aus der Macht der Wiederholung zu befreien, würde der „Unmöglichkeit ein Spalt geschaffen“, ein Moment des „Möglichwerden[s] einer Unmöglichkeit, die [...] erst dann [...] gewählt und aktualisiert werden kann“" (S. 214).

Die Argumentation des Buches eröffnet reiche Möglichkeiten zur Diskussion unter dem leitenden Motiv der Wiederholung, auch wenn man der Autorin nicht in allem folgen mag, indem die von ihr entwickelte Argumentation aufgegriffen und Elemente davon wiederholend variiert werden können, um zu sehen, welche Denkmöglichkeiten dabei erscheinen. Um zwei Beispiele zu geben: Nietzsches Frage, wie ich über meine Handlungen verfügen könne, wenn doch alles notwendig sei (S. 110), lässt sich anders beantworten, wenn von der starren mechanischen Kräftelehre auf das Konzept von (kultureller) Evolution übergegangen wird. Zukunft ist dann prinzipiell offen und definitiv ohne Ziel, die Wiederkehr des ewig Gleichen ist ein Effekt bestimmter Systemzustände, nicht der Welt als Ganzer. Dennoch können auch hier Trajektorien des Möglichkeitsraumes nicht beliebig gewählt, oder vorher beschrittene beliebig verlassen werden. Akteure sind weiterhin an die Möglichkeiten gebunden, die ihnen der gegenwärtige Systemzustand zur Verfügung stellt. Unter dem Konzept der Evolution könnten problematisch erfahrene gesellschaftliche Entwicklungen zwar immer noch auf traumatische Ereignisse zurückgeführt werden, es ist aber auch denkbar, dass Entwicklungen, wie die Einführung von Privateigentum 
(S. 99f.), in der entsprechenden Entwicklungsphase als Lösung für ein Problem und nicht als Trauma erlebt worden sind, oder doch zumindest als ,eine gute Idee', woraus sich auch ihr Beharrungsvermögen erklären ließe.

Löschenkohls Argumentation begründet weniger konkrete Handlungsweisen als vielmehr eine bestimmte Haltung gegenüber der eigenen Begrenztheit und der eigenen Fehlbarkeit angesichts hochkomplexer Herausforderungen. Eine gewisse Gefahr besteht angesichts der (zu starken?) Betonung des Begriffes der Unmöglichkeit des Handelns und der Unverfügbarkeit der Zielsetzung emanzipatorischer Bewegungen darin, die Haltung zu sehr aus Richtung des die Last akzeptierenden Kamels Nietzsches einzunehmen als aus der Perspektive des schaffenden, spielenden Kindes (S. 122f. und 147). Henning Ottmann hatte in „Philosophie und Politik bei Nietzsche“ (1987) den Gedanken eingebracht, Nietzsches Philosophie mit dem amerikanischen Pragmatismus in Diskussion zu bringen. Tatsächlich bietet John Deweys Konzeption des „Erfahrungen-Machens“, zu dem auch das Scheitern gehört, das in die eigene Geschichte integriert werden kann, angesichts eines nicht vollumfänglich bestimmten „Ends-in-View“ aus „Kunst als Erfahrung“ (1988) ein Korrektiv zu der genannten Gefahr und eine gute zusätzliche Lektüre zu Birte Löschenkohls höchst lesenswertem und bereicherndem Buch.

Funding Open Access funding provided by Projekt DEAL.

Open Access Dieser Artikel wird unter der Creative Commons Namensnennung 4.0 International Lizenz veröffentlicht, welche die Nutzung, Vervielfältigung, Bearbeitung, Verbreitung und Wiedergabe in jeglichem Medium und Format erlaubt, sofern Sie den/die ursprünglichen Autor(en) und die Quelle ordnungsgemäß nennen, einen Link zur Creative Commons Lizenz beifügen und angeben, ob Änderungen vorgenommen wurden.

Die in diesem Artikel enthaltenen Bilder und sonstiges Drittmaterial unterliegen ebenfalls der genannten Creative Commons Lizenz, sofern sich aus der Abbildungslegende nichts anderes ergibt. Sofern das betreffende Material nicht unter der genannten Creative Commons Lizenz steht und die betreffende Handlung nicht nach gesetzlichen Vorschriften erlaubt ist, ist für die oben aufgeführten Weiterverwendungen des Materials die Einwilligung des jeweiligen Rechteinhabers einzuholen.

Weitere Details zur Lizenz entnehmen Sie bitte der Lizenzinformation auf http://creativecommons.org/ licenses/by/4.0/deed.de. 